\title{
Combined D-optimal design and generalized regression neural network for modeling of plasma etching rate
}

\author{
Hailong You ${ }^{1,2 \star}$, Yong $\mathrm{Chen}^{3}$, Peng $\mathrm{Liu}^{1,2}$, and Xinzhang $\mathrm{Jia}^{1,2}$ \\ ${ }^{1}$ School of Microelectronics, Xidian University, Xi'an 710071, P.R. China \\ 2 Key Laboratory of Wide Band-Gap Semiconductor Materials and Devices, Xi'an 710071, P.R. China \\ 3 School of Economics and Management, Xidian University, Xi'an 710071, P.R. China
}

Received: 6 November 2013 / Accepted: 24 April 2014

\begin{abstract}
Plasma etching process plays a critical role in semiconductor manufacturing. Because physical and chemical mechanisms involved in plasma etching are extremely complicated, models supporting process control are difficult to construct. This paper uses a 35-run D-optimal design to efficiently collect data under well planned conditions for important controllable variables such as power, pressure, electrode gap and gas flows of $\mathrm{Cl}_{2}$ and $\mathrm{He}$ and the response, etching rate, for building an empirical underlying model. Since the relationship between the control and response variables could be highly nonlinear, a generalized regression neural network is used to select important model variables and their combination effects and to fit the model. Compared with the response surface methodology, the proposed method has better prediction performance in training and testing samples. A success application of the model to control the plasma etching process demonstrates the effectiveness of the methods.
\end{abstract}

Keywords: D-optimal design, generalized regression neural network, response surface methodology, plasma etching rate

\section{Introduction}

As the semiconductor manufacturing moves forward nanofabrication, the variability of device and circuits parameters induced by fabrication equipment and environment becomes an important problem affecting IC quality and yield improvement [1]. Many approaches on characterizing, controlling and optimizing of complex processes have been developed to control the variability and maximize the process yield over the years $[2,3]$. Response surface methodologies and neural networks are usually used to construct the empirical model that describes the relation of the critical process response with the important input parameters $[2,3]$. Then the manufacturing process is optimized and controlled using these empirical models.

Nowadays, as plasma etching process is the mostly used method to transfer pattern in semiconductor manufacturing, it plays a key role in the variability control and the yield improvement of devices and circuits. Researchers have developed models for plasma etching process using physical theory, statistical design of experiment, and neural network, etc. Because modern plasma etching process involves physical and chemical mechanisms, theoretical modeling based on principles is difficult to construct. In addition, it has some limit in the process control and optimization application, especially processes real-time control due to the model size and complexity [4-6]. So as

^Correspondence: hlyou@mail.xidian.edu.cn an alternative to first principle models, empirical models based on statistical design of experiment plays an important role in plasma etching control and optimization [7]. In the research on statistical modeling of semiconductor process, neural network has shown more advantages than response surface methodology (RSM) for plasma etching in accuracy and robustness. In recent years, various network structures have been applied in plasma etching modeling $[8,9]$. For example, Kim and Park used RBFN for the plasma etching process modeling [9-11]. Compared to traditional response surface methodology, the neural network model based on RBFN increased more than $40 \%$ in the prediction ability of etching rate.

The choice of experimental design and neural networks structure is a key problem in statistical modeling of plasma etching process. In this paper, a generalized regression neural network combined with D-optimal design is used to modeling the etching rate in the plasma etch process. The rest of the paper is organized as follows. Section 2 describes the plasma etching process and equipment. Experimental design and experiment are discussed in Section 3. Detailed modeling process is provided in Section 4. The last section is the conclusion.

\section{Plasma etching equipment and process}

A schematic diagram of an experimental plasma etch system is shown in Figure 1. The etching apparatus consists 
Table 1. Experimental factors, ranges and levels.

\begin{tabular}{ccc}
\hline Factors & Ranges & Experimental levels \\
\hline Power (A) & $(100,300) \mathrm{w}$ & $(100,200,300) \mathrm{w}$ \\
Pressure (B) & $(250,450) \mathrm{mT}$ & $(350,400,450) \mathrm{mT}$ \\
Electrode Gap $(\mathrm{C})$ & $(0.5,1.5) \mathrm{cm}$ & $(0.5,1,1.5) \mathrm{cm}$ \\
$\mathrm{Cl}_{2}$ Flow $(\mathrm{D})$ & $(50,100) \mathrm{sccm}$ & $(50,66.66,83.84,100) \mathrm{sccm}$ \\
He Flow (E) & $(100,200) \mathrm{sccm}$ & $(100,133.33,166.66,200) \mathrm{sccm}$ \\
\hline
\end{tabular}

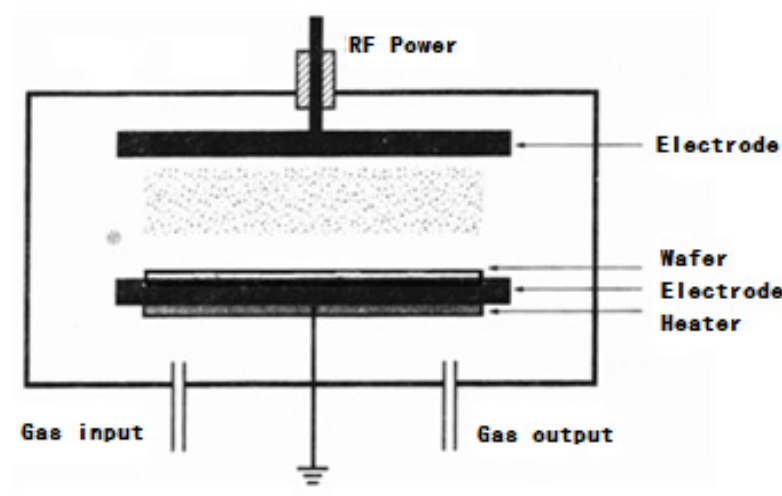

Fig. 1. Schematic diagram of plasma etching equipment.

of a single-wafer parallel-plate system. The chamber pressure is maintained in 100 mtorr to 1 torr. The etching samples rest on the grounded lower electrode while the upper electrode is excited by a $13.56 \mathrm{MHz} \mathrm{RF}$ generator operating through a matching network. In this experiment the plasma etching equipment is used as a fabrication step in the definition of polysilicon features for MOS circuit. The quality of this fabrication process is usually characterized by its selectivity, anisotropy, uniformity, and etching rate.

Etching rate is the most critical response in plasma etching process. Because poly is the main material etched and the relatively thick polysilicon gate be etched down to a thin silicon dioxide layer in the process of the polysilicon feature definition, the vertical etching rate of polysilicon is important response. The control of polysilicon etching rate is critical for the determination of etching stop point. The modeling of polysilicon etching rate is discussed in this paper. Etching was performed on a simple test structure designed to facilitate the simultaneous measurement of the vertical etching rates of polysilicon, $\mathrm{SiO}_{2}$, and photoresist. The cross section showing the critical measurement area is shown in Figure 2. Vertical etching rate is calculated by dividing the difference between pre-etch and post-etch thickness by etch time. The polysilicon etching rate is defined as equation (1).

$$
R=\Delta d / t
$$

where $\Delta d$ is the thickness difference of the film between pre-etch and post-etch, and $t$ is the etching time.

The other responses are generally represented by etching rate directly or indirectly. For example, the selectivity of the polysilicon with respect to the oxide is defined as follows:

$$
S=\frac{R_{p}}{R_{o x}}
$$

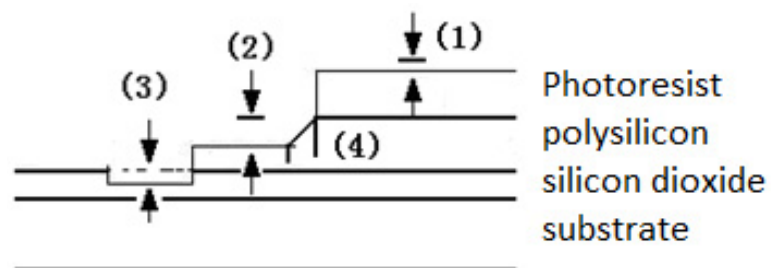

Fig. 2. Test pattern in plasma etching process.

where $R_{p}$ is the mean vertical poly etch over the five points, and $R_{o x}$ is the mean oxide etching rate.

The critical input parameters in plasma etching are RF power, chamber pressure, electrode gap, and gas flow. Inert gas, such as Helium in this experiment, is often added to standard $\mathrm{Cl}_{2}$ etch recipes in order to enhance etch uniformity. Helium flow has an important effect on an anisotropy. Therefore these six input parameters including $\mathrm{RF}$ power, reactor pressure, electrode gap, $\mathrm{Cl}_{2}$ flow rate and He flow rate should be considered in modeling of polysilicon etching rate.

\section{Experimental design and D-optimal design}

\subsection{Experimental factors and respective chosen levels}

Five process input parameters shown in Table 1 are chosen as input variables in modeling of the plasma etch process. Modeling of plasma etching process revealed significant nonlinearity in all responses in recently year. Quadratic effects of input parameters should be considered. In addition, based on plasma etching theory and experience of process engineer, different input factors are different in importance for polysilicon etching rate. In polysilicon etching process, $\mathrm{Cl}_{2}$ is the reactant gases, and Helium can improve the uniform and anisotropy. These two parameters are more important than others. Therefore, four levels in ranges of $\mathrm{Cl}_{2}$ and Helium gas flow are chosen, while three levels for RF power, chamber pressure, and electrode gap. Table 1 shows the input parameters, their respective range, and the chosen levels in the modeling experiment.

\subsection{D-optimal design and experimental plan}

The statistical modeling is usually based on design of experiment data, as well as neural network. How to design the experiment plan is critical for modeling of etching rate. The collected data according to the experiment plan generated by DOE should completely represent the properties of the process. However, the DOE data is limited as experiment costs a lot. In addition, the experiment plan should 
avoid events that should not be performed in the experiment because of the limitation of the equipment ability. For example, the plasma etching equipment in this research cannot run in condition of low chamber pressure combined with high gas flow.

Traditional experimental design like factorial design and response surface design do not meet the situation. In this paper the D-optimal design was explored to generate the experiment plan because of its following unique features:

(1) D-optimal design allows for a flexible number of experiments, depending on the experimental cost.

(2) D-optimal design can deal with the different levels of experiment factors.

(3) Through constraint condition in optimization process of D-optimal design is chosen based on the equipment control ability, the experiment plan generated by D-optimal design can avoid the runs that cannot carry on in the equipment.

(4) Because D-optimal design is best suited for the known model structure, the knowledge of the physical theory and process engineers on the equipment and process can be considered in the experimental design process.

In order to generate a D-optimal design for plasma etching process in this study, the etching rate model structure described as following is used:

$$
\begin{aligned}
y= & \beta_{0}+\sum_{k=1}^{5} \beta_{k} x_{k}+\sum_{j=1}^{3} \sum_{k=j}^{3} \beta_{j k} x_{j} x_{k} \\
& +\beta_{445} x_{4}^{2} x_{5}+\beta_{444} x_{4}^{3}+\beta_{455} x_{4} x_{5}^{2}+\beta_{555} x_{5}^{3},
\end{aligned}
$$

where $x_{i}$ represents the input variables, and $\beta_{i}$ represents the model coefficient.

The D-optimal design criterion is used to choose the runs from the set of $432\left(3^{*} 3^{*} 3^{*} 4 * 4\right)$ possible experimental combinations. Considering the experimental budget and the data requirement of modeling, the experimental plan including 35 runs is generated from the possible experiment combinations.

\section{Modeling of plasma etching rate}

Neural networks have been popular in semiconductor process modeling because of their ability to learn complex nonlinear system. Plasma etching process involves physical and chemical theory. In past work on modeling of plasma etching process, it has been shown that neural networks offer superior modeling capabilities as compared to statistical techniques. However, neural networks have various structures and training algorithms used to implement neural network learning. Model accuracy is greatly influenced by the structures and training algorithms. In neural network modeling, back-propagation neural network (BPNN) and radial basis function neural network (RBNN) are frequently used for modeling of plasma etching process. But BPNN and RBF model are too complicated for many training factors. In addition, the experiment data are limited due to the experiment cost and the

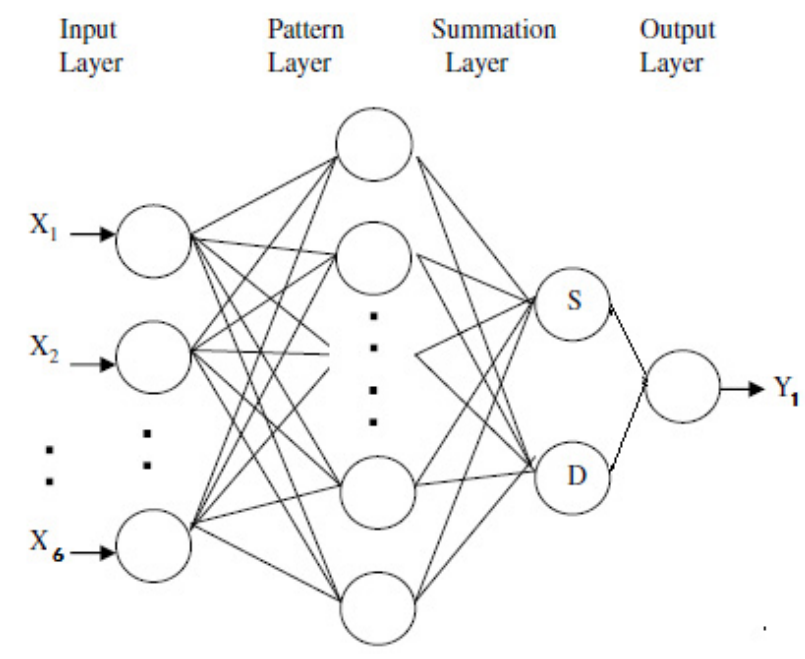

Fig. 3. A schematic of GRNN structure.

plasma etching process complexity. The prediction ability of model trained by the same data based on the various network structures may be significantly different. For the specific experiment data, it is enough for some networks, but is not for others. BPNN and RBF usually need more train samples than a generalized regression neural network [12]. Therefore, for the plasma etching equipment and process in this research, a generalized regression neural network is adopted to learn process response from 35 samples data generated by D-optimal design without prior knowledge.

\subsection{GRNN structure and parameters setting}

Generalized regression neural network was proposed by Specht as an alternative to back-error propagation training algorithm for feedforward neural networks. A general structure of the GRNN can be illustrated as in Figure 3. As it can be seen from Figure 3, the GRNN consists of three layers of nodes: the input layer, the hidden layer (including the pattern layer and the summation layer), and the output layer.

The input layer translates the input parameters in training sample sets to the GRNN. So each input unit in the input layer respectively corresponds to input parameters. Each unit in the pattern layer represents a training pattern. The summation layer will perform the summing operation as for the upper and lower parts. The final output of network is obtained at the output layer. For the experiment data sets including six input parameters, one output response and 35 runs data, the number of input units, pattern units and output units are 6, 35 and 1, respectively.

\subsection{Modeling of plasma etching rate}

The model is constructed by using the Matlab neural network toolbox. The model parameters for GRNN include the numbers of input layer, hidden layer neurons and the SPREAD, where SPREAD is chosen by an operator for 
Table 2. Comparison of GRNN and Response surface models prediction performance.

\begin{tabular}{cccccc}
\hline Run & $\begin{array}{c}\text { Experiment } \\
\text { result }\end{array}$ & $\begin{array}{c}\text { Prediction of } \\
\text { GRNN model }\end{array}$ & $\begin{array}{c}\text { Prediction of response } \\
\text { surface model }\end{array}$ & $\begin{array}{c}\text { Prediction error } \\
\text { of GRNN model (\%) }\end{array}$ & $\begin{array}{c}\text { Prediction error of response } \\
\text { surface model }(\%)\end{array}$ \\
\hline 1 & 3251.40 & 3151.55 & 2380.32 & 3.07 & 24.47 \\
2 & 3458.20 & 3481.90 & 3162.27 & 0.69 & 9.18 \\
3 & 4006.20 & 3335.08 & 2937.77 & 16.75 & 11.91 \\
4 & 5200.00 & 5289.43 & 5043.60 & 1.72 & 4.65 \\
5 & 4129.00 & 3802.68 & 3856.27 & 7.90 & 1.41 \\
\hline \multicolumn{7}{c}{ Average prediction errors (\%): } \\
\hline
\end{tabular}

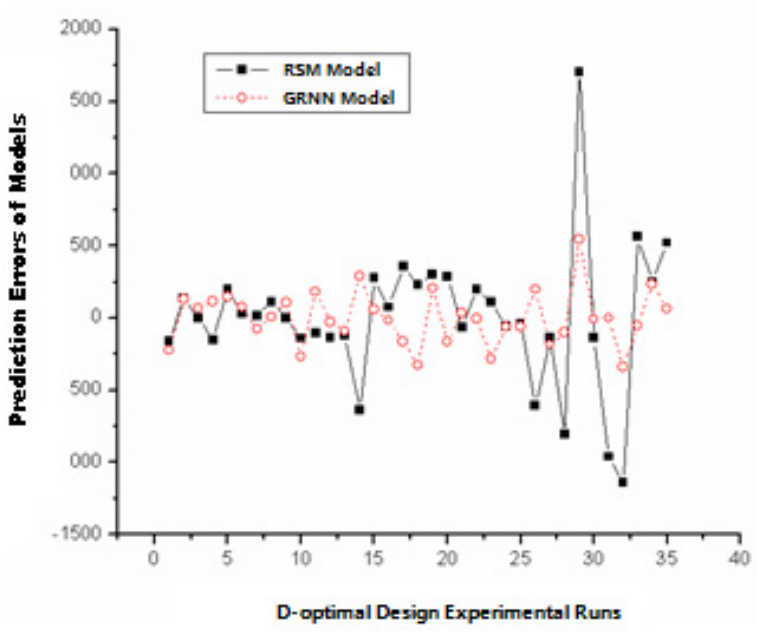

Fig. 4. Comparison of prediction errors between GRNN model with RSM model.

high predicting ability. The centers of the Gaussian are chosen equal to the training input patterns. Each hidden node has an associate bias that plays the role of the variance of Gaussian. The bias is set to a column vector of $0.8326 / \mathrm{SPREAD}$. For modeling of plasma etching rate with 35 runs date set, the structure of the generalized regression neural network consist of six units in the input layer, 35 units in pattern layer, 2 units in the summary layer and one unit in the output layer as described in Figure 3. Therefore, SPREAD is the only one network parameter needed to be regulated. It can be obtained using solving the problem of minimizing the root mean square error for the GRNN model prediction of training set. Through linearly searching in range of 0.1 to 100 , the error is minimized when the value of SPREAD is about 90. So for GRNN modeling of the plasma etch process, SPREAD is chosen as 90 .

Based on the experiment data, the GRNN model is constructed. In addition, response surface methodology (RSM) is used to construct the model based on the same data. Figure 4 shows the two models fitting errors of the prediction with the experiment result. As can be seen in Figure 4, the fitting errors of GRNN model is are much smaller than that of the statistical regression model.

The fitting performance is measured by the fitting ratio of signal to noises defined as:

$$
S N R=10 \log \frac{\operatorname{En}(d)}{\operatorname{En}(e)},
$$

where $\operatorname{En}()$ represents the sum of squares, $e$ is the fitting errors, $e=d-f$;

$$
E n(d)=\sum_{i=0}^{n-1} d^{2}, \quad E n(e)=\sum_{i=0}^{n-1} e_{i}^{2}
$$

the greater the fitting SNR is, the better fitting ability the model has. The fitting SNR of The GRNN model and regression model are 62.99 and 43.97 , respectively. Therefore, the fitting ability GRNN model is better than the statistical regression model.

\subsection{Prediction performance of models}

The GRNN model of plasma etching rate can be used to predict the response in the different input conditions. In the area around the possibility optimal process input, five input combinations are chosen randomly to evaluate the prediction ability of the model. The results of the model prediction and experiment are shown in Table 2. Table 2 also show the prediction result of the statistical regression model. The mean prediction errors of the GRNN and statistical regression models are $6.03 \%$ and $10.32 \%$, respectively. Compared to the statistical regression model, the plasma etching rate GRNN model shows much improvement in the prediction ability.

The model with an average prediction error of $6 \%$ is used in the process control. With this model, the etching end point, which is directly related to etching time, can be obtained for the specified etching thickness in the different input conditions. In addition, the process can be diagnosed whether it works normally through the comparison of the model prediction with the actual result.

\section{Conclusion}

This paper discussed an effective method using D-optimal design combined with generalized regression neural network for plasma etching process modeling. Considering the various role of six input parameters (including the power, the pressure, the gap and the gas flow of $\mathrm{Cl}_{2}, \mathrm{He}$ ) and underlying model forms in plasma etching based on theoretical knowledge and engineer experience, D-optimal design is adopted to generate the sampling plan. Based on the experiment data, generalized regression neural network is used to develop the model of the plasma etching rate which 
H. You et al.: Combined D-optimal design and generalized regression neural network for modeling of plasma... 105-p5

is the most important output response in plasma etching process. Compared to the response surface model, the GRNN model achieves a better prediction performance. The model constructed by the method has been successful applied in the plasma etching process control and the end point prediction of etching process.

Acknowledgements. Project supported by the National Natural Science Foundation of China (Grant No. 60906051).

\section{References}

1. H. Ramakrishnan et al., Analysing the effect of process variation to reduce parametric yield loss, IEEE International Conference on Integrated Circuit Design and Technology and Tutorial, ICICDT. Austin, TX, USA, 2008

2. P. Sung-Woo et al., Microelectron. Eng. 66, 488-495 (2003)
3. L. Hsin-Te, S. Jie-Ren, Y. Yung-Kuang, Int. J. Adv. Manufact. Technol. 38, 674-682 (2008)

4. Z.N. Mevawalla, G.S. May, M.W. Kiehlbauch, Neural networks for advanced process control, in Advanced Semiconductor Manufacturing Conference (ASMC), IEEE/SEMI, San Francisco, CA, USA, 2010

5. Y.H. Long, J.X. Zhang, Chin. J. Electron. 16, 69-72 (2007)

6. K. Byungwhan, J.K. Bae, W.-S. Hong, J. Vac. Sci. Technol. A 23, 355-358 (2005)

7. D. Stokes, G.S. May, IEEE Trans. Semicond. Manufact. 13, 469-480 (2000)

8. S.J. Hong, G.S. May, P. Dong-Cheol, IEEE Trans. Semicond. Manufact. 16, 598-608 (2003)

9. K. Byungwhan et al., J. Appl. Phys. 105, 113302 (2009)

10. S.J. Hong, G.S. May, IEEE Trans. Semicond. Manufact. 17, 408-421 (2004)

11. B. Kim, D. Lee, K.H. Kwon, J. Appl. Phys. 96, 3612-3616 (2004)

12. B. Kim, K. Park, Microelectron. Eng. 77, 150-157 (2005)

13. D.F. Specht, IEEE Trans. Neural Networks 2, 568-576 (1991) 